\title{
Optical Properties of self-assembled InGaAs Quantum wires grown on (100) GaAs Substrate
}

\author{
Jong-Horng Dai, Yi-lung Lin, and Si-Chen Lee \\ Graduate Institute of Electronics Engineering, National Taiwan University, Taipei, Taiwan, 10617 \\ Shih-Yen Lin and Jim-Yong Chi \\ Nanophotonic Center, Opto-Electronics \& Systems Labortories, Industrial Technology Research \\ Institute, Taiwan
}

\begin{abstract}
A method is proposed to grow InGaAs quantum wires on (100) GaAs substrates by solid-source molecular beam epitaxy via Stranski-Krastanov growth mode. The shape of the nano-wire structures are measured by the atomic force microscopy (AFM). Their optical properties are studied by temperature-dependent photoluminescence (PL). The integrated PL intensity at room temperature reduced to $10 \%$ of that at $20 \mathrm{~K}$. From 20 to 300 $K$, the PL peak energy shifts only $70 \mathrm{meV}$.
\end{abstract}

Index Terms - AFM, GaAs substrate, Nano structure, Potoluminescence, Quantum wire, Solid- source MBE

\section{INTRODUCTION}

Low-dimensional semiconductor structures such as zero-dimensional quantum dots (QD), one-dimensional quantum wires (QWR) and two-dimensional quantum wells $(\mathrm{QW})$ were well known in quantum heterostructures in which electrons are confined in one or more dimensions, but are free in the other dimensions. Quantum dots and quantum wires have already been made by mature growth techniques and have many applications. Although there are many fabrication techniques such as e-beam, micro lithograph or laser to form one dimensional QWR [1]-[3], it is difficult to get QWR on normally oriented (100) GaAs substrate. In recent years, there are many reports that anisotropic quantum dots (wire-like QDs) could be formed on misoriented (001) InP, vicinal (001) GaAs and (311)-oriented GaAs substrates [4]-[6]. In this paper, we present a method to grow InGaAs quantum wires on norminally oriented (100) GaAs substrate. The samples were characterized by atomic force microscopy (AFM). Temperature dependent photoluminescence (PL) exhibits the ground state emission. The PL intensities and peak energies vary with temperature. The results are correlated with the optical anisotropy of the nano-wire as indicated by polarized photoluminescence (PPL).

\section{EXPERIMENTS}

The sample was grown by Riber Epineat solid-source molecular beam epitaxy (MBE) on (100) GaAs substrate. The substrate temperature was measured using an infrared pyrometer. After thermal desorption of native oxide on the GaAs substrates, a 500-nm GaAs buffer layer was grown. The growth temperature was fixed at 600 . The growth rate is $1 \mu \mathrm{m} / \mathrm{h}$. Then the temperature of the substrate is lowered to 510 , and a $2.4 \mathrm{ML}$ of InAs were grown at a $0.1 \mathrm{ML} / \mathrm{s}$ growth rate. The arsenic pressure was fixed at $7 \times 10^{-7}$ Torr. InAs QDs are formed on the GaAs buffer layer. After InAs deposition, the sample was capped with $3 \mathrm{~nm} \mathrm{GaAs}$ at the same temperature. Then the growth was interrupted for $30 \mathrm{sec}$ under same As pressure. The sample was subsequently cooled, at a cooling rate of $20 / \mathrm{s}$, to room temperature and removed from the system.

Sample was scanned by a NT-MDT Instruments atomic force microscope (AFM) operated in the tapping mode. In order to characterize the carrier confinement in these quantum wires by PL measurements, the samples were capped with a $60 \mathrm{~nm}$ GaAs layer. The PL spectra were obtained in the $20-300 \mathrm{~K}$ temperature range and excited using the $514.5 \mathrm{~nm}$ line of argon laser. The power dependent photoluminescence (PL) spectra of quantum wires with power changing from 50 to $400 \mathrm{~mW}$ were measured at $20 \mathrm{~K}$. For polarization photoluminescence (PPL) measurements the [0-11] direction of the samples is oriented at $45^{\circ}$ with respect to the polarization of the laser to get both balanced pumping along [0-11] and [011] directions. A polarizer at the entrance slit of the monochromator is used to measure PL intensity along both directions. The linear polarization degree $\mathrm{P}$ defined as ( [0-11]-[011]) / ([0-11] $+[011])$ of the emitted light. As a reference a QD sample was grown at the same 
conditions but without the $3 \mathrm{~nm}$ GaAs layer partially capping the dots.

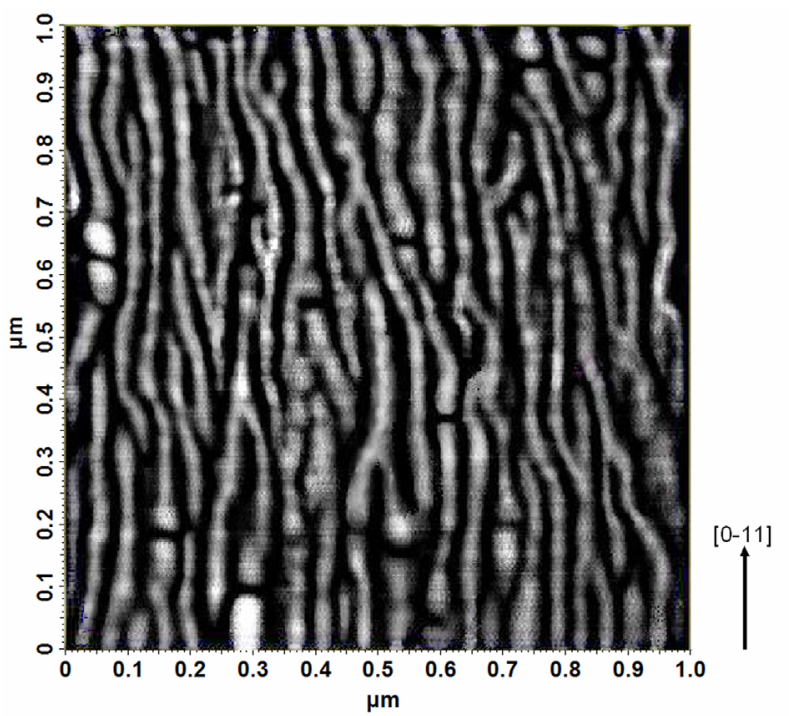

(a)

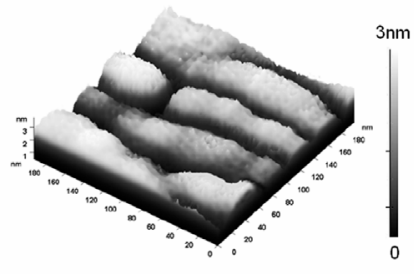

(b)

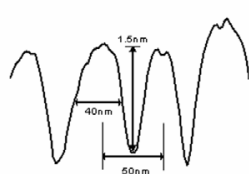

(c)
Fig. 1. (a) $1 \mathrm{um} \times 1 \mathrm{um}$ AFM images of quantum wires. (b) $200 \mathrm{~nm} \times 200 \mathrm{~nm}$ in $3 \mathrm{D}$ image and (c) the cross section profile taken from (b).

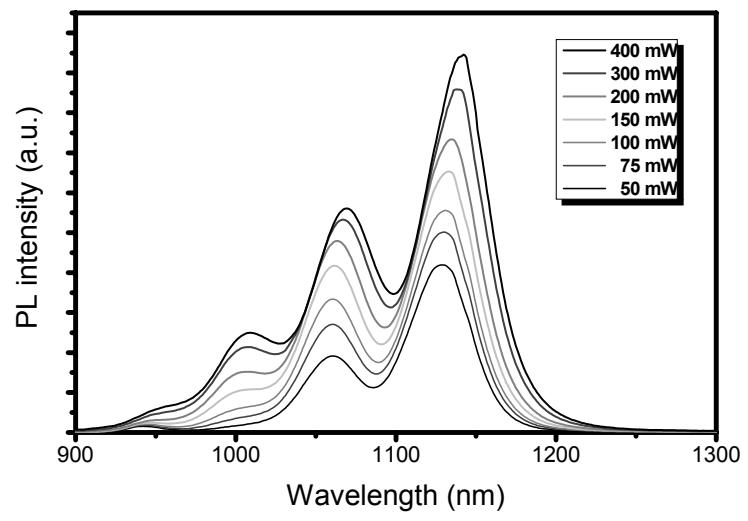

Fig. 2. PL of InGaAs quantum wires under different excitation power. All the measurements were performed at $\mathrm{T}=20 \mathrm{~K}$.

\section{RESULTS AND DISCUSSION}

Fig. 1 shows the AFM images of quantum wires. Wire shaped structure oriented along the [0-11] direction is observed in Fig. 1(a). The 3D image and cross section profile was shown in Fig. 1(b) and (c). After stretching them out, some of them may bumped into each other; some may bifuracate or break. But quantum wires still keep in [0-11] direction. The average width is $40 \mathrm{~nm}, 1.5$ $\mathrm{nm}$ in height. The length of the wire is between 300 to 600 $\mathrm{nm}$.

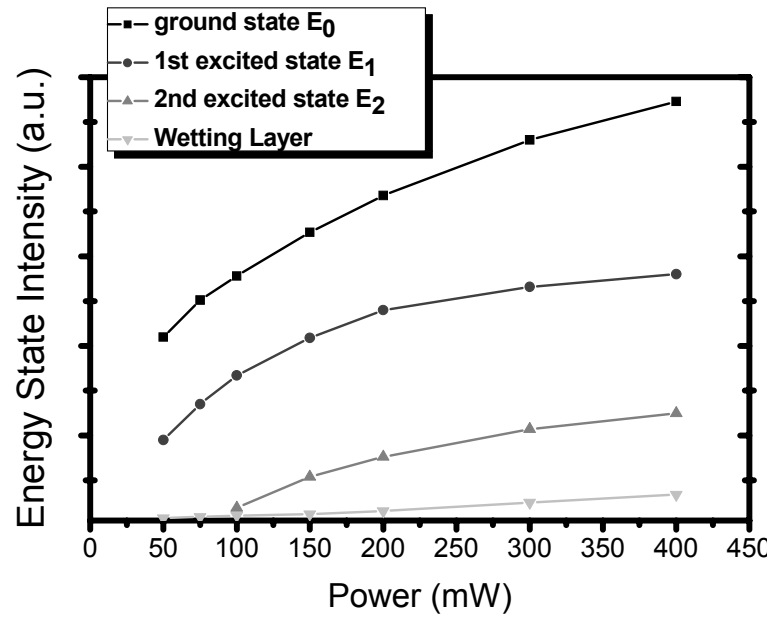

Fig. 3. Four PL peak intensities increase with laser power density.

Fig. 2 shows the PL spectra at 20K. There are 3 major peaks plus a small wetting layer emission peak. The PL spectra were taken under different excitation power from 50 to $400 \mathrm{~mW}$. Four peaks increased with the laser power. From Fig. 3, each peak intensity increases with different slope. The four peaks with different strength located at $1.094 \mathrm{eV}$ (ground state peak $\mathrm{E}_{0}$ ), $1.168 \mathrm{eV}$ (first excited state peak $\mathrm{E}_{1}$ ), $1.233 \mathrm{eV}$ (second excited state peak $\mathrm{E}_{2}$ ), $1.309 \mathrm{eV}$ (wetting layer peak WL). Fig. 4. shows temperature dependence of the PL peak positions of the QWR ground state, excited state and QD ground state from 20 to $300 \mathrm{~K}$. The QD and QWR peak energies are both shifted $70 \mathrm{meV}$. The PL intensity of the QWR at room temperature remains $10 \%$ of the PL intensity at $20 \mathrm{~K}$.

Fig. 5 shows the PL peak associated with quantum wires which exhibits a large anisotropy for the orthogonal [0-11] and [011] polarization with a peak intensity ratio of 1.82. The degree of linear polarization for the quantum wires samples is near $28 \%$. 


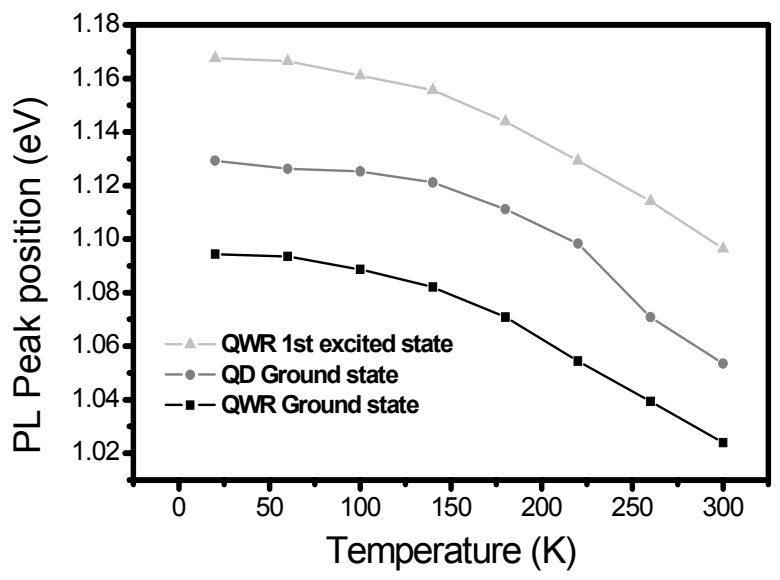

(a)

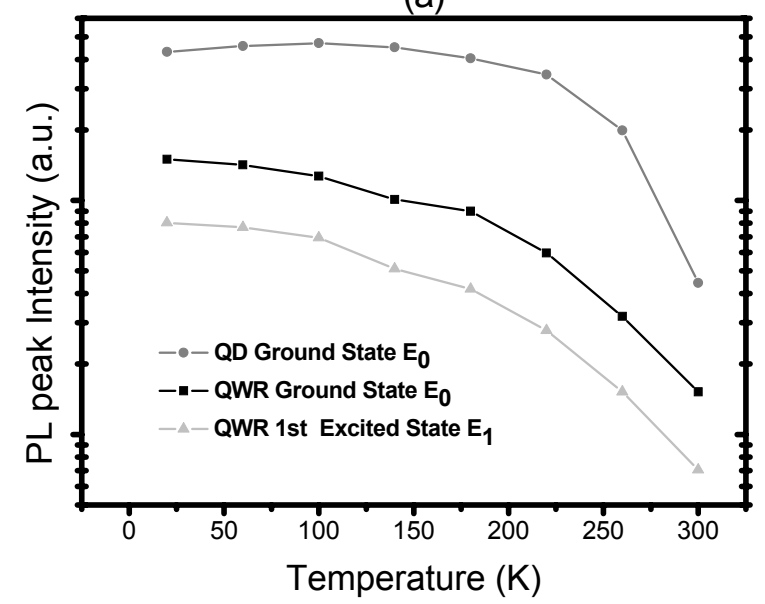

(b)

Fig. 4. Temperature dependence of the PL peak (a) energy; (b) intensiy for quantum wires compared with quantum dots samples.

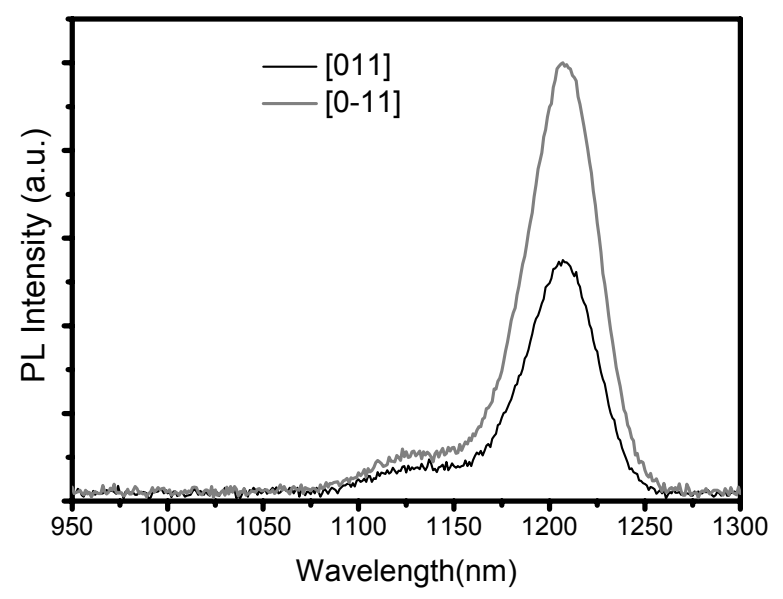

Fig. 5. RT polarized PL spectra of the quantum wires sample observed for [011] and [0-11] polarization.

\section{CONCLUSION}

From this study, it is found that a wire-like shape of self-assembled nanostructures of $\mathrm{InGaAs} /(100) \mathrm{GaAs}$ can be formed without any regrowth or patterned process. The optical properties of the quantum wire were observed from PL spectra which satisfied quantum confinement of nano structure. Additional information about the temperature dependence of the integrated PL peak energy and intensity has stable characteristics that remain very strong at room temperature for infrared optoelectronic device. Besides using quantum dot structures, quantum wire devices on the GaAs substrate can be realized.

\section{ACKNOWLEDGEMENT}

The authors are grateful to C. Y. Liang for helpful discussion about PL measurment.

\section{REFERENCES}

[1] S. J. Rehse, R. W. McGowan, and S.A. Lee, "Optical manipulation of group III atoms" Appl. Phys. B, vol. 70, pp. 657-660, April 2000.

[2] Hideaki Saito, K. Nishi, S. Sugou, and Y. Sugimoto, "Controlling polarization of quantum-dot surface-emitting lasers by using structurally anisotropic self-assembled quantum dots" Appl. Phys. Lett, vol. 71, no. 5, pp. 590-592, August 1997.

[3] M. Takahashi, P. Vaccaro, K. fujita, T. Watanabe, T. Mukaihara, F. Koyama, and K. Iga, "An InGaAs-GaAs Vertical-Cavity Surface-Emitting Laser Grown on GaAs(311)A Substrate Having Low Threshold and Stable Polarization" IEEE Photonic. Tech. Let., vol. 8, no. 6, pp. 737-739, June 1996.

[4] M. Henini, S. Sanguinetti, S. C. Fortina, E. Grilli, and M. Guzzi, M. D. Upward, P. Moriarty, P. H. Beton, and L. Eaves "Optical anisotropy in arrow-shaped InAs quantum dots" Phys. Rev. B, vol. 57, no. 12, pp. R6815-R6818, March 1998.

[5] J. Brault, M. Gendry, G. Grenet, G. Hollinger, J. Olivares, B. Salem, T. Benyattou, and G. Bremond, "Surface effects on shape, self-organizatioin and photoluminescence of InAs islands grown on InAlAs/InP(001)" J. Appl. Phys., vol. 92, no. 1 , pp. 506-510, July 2002.

[6] J. Brault, M. Gendry, O. Marty, M. Pitaval, J. Olivares, G. Grenet, and G. Hollinger, "Staggered vertical selforganization of stacked InAs/InAlAs quantum wires on InP(001)" InAlAs/InP(001)" Appl. Surf. Sci., vol. 162-163, pp. 584-589, 2000. 
